\title{
OBSERVATIONS OF EXTRAGALACTIC RADIO EMISSION
}

\author{
C. A. SHAIN \\ Radiophysics Laboratory, Commonwealth Scientific \\ and Industrial Research Organization, Sydney, Australia
}

1. INTRODUCTION

Several extragalactic radio sources within reach of Sydney radio telescopes have been found to have angular sizes so great that they can be resolved with the pencil-beam aerials, which are available at three frequencies. It is possible to study in some cases the brightness distribution in some detail; in others to study the qualitative general picture. The observations with which the present paper is concerned are set out in Table I.

TABLE I

\section{ReCEnt Sydney ObServations of Extragalactic Sources}

$\begin{array}{llll}\text { Source } & \begin{array}{c}\text { Associated } \\ \text { Galaxy }\end{array} & \begin{array}{c}\text { Observing } \\ \text { frequency (Mc/s) }\end{array} & \text { Remarks } \\ \text { Centaurus A } & \text { NGC } 5128 & 19.7,85.5,1390 & \text { Detailed isophotes } \\ \text { Fornax A } & \text { NGC } 1316 & 19.7,85.5 & \text { General picture } \\ \text { Virgo A } & \text { NGC } 4486 & 19.7,85.5 & \text { General picture } \\ & \text { L.M.C. } & 19.7 & \text { Detailed isophotes } \\ & \text { S.M.C. } & 19.7 & \text { General picture }\end{array}$

Aerials: $19.7 \mathrm{Mc} / \mathrm{s}, 1.4 \mathrm{cross} ; 85.5 \mathrm{Mc} / \mathrm{s}, 0.8$ cross; $1390 \mathrm{Mc} / \mathrm{s}, 1.5$ paraboloid.

\section{OBSERVATIONS}

\section{(a) Centaurus $A$}

Preliminary observations of this source at $85.5 \mathrm{Mc} / \mathrm{s}$ were described at the Jodrell Bank Symposium by de Vaucouleurs and Sheridan [1]. Since then, the observational data have been improved, and after a more refined analysis Sheridan [2] has prepared a final set of isophotes which are shown in Fig. $1(a)$. Isophotes for a frequency of $19.7 \mathrm{Mc} / \mathrm{s}$ are now available [3], and these are given in Fig. $1(b)$. At both frequencies the general pictures are similar: an elongated source with a bright concentration near the center. The central source stands out much more clearly at $85.5 \mathrm{Mc} / \mathrm{s}$; this is partly, but not wholly, an effect of the greater aerial gain at this frequency. In the course of an observational program, which is still continuing, C. M. Wade and J. V. Hindman have found that even at $1390 \mathrm{Mc} / \mathrm{s}$ the elongated component of the source can be readily detected, although the extension is probably not as great as at the lower frequencies. On the other hand, the subsidiary maximum at about declination -45 degrees is rather more prominent. 

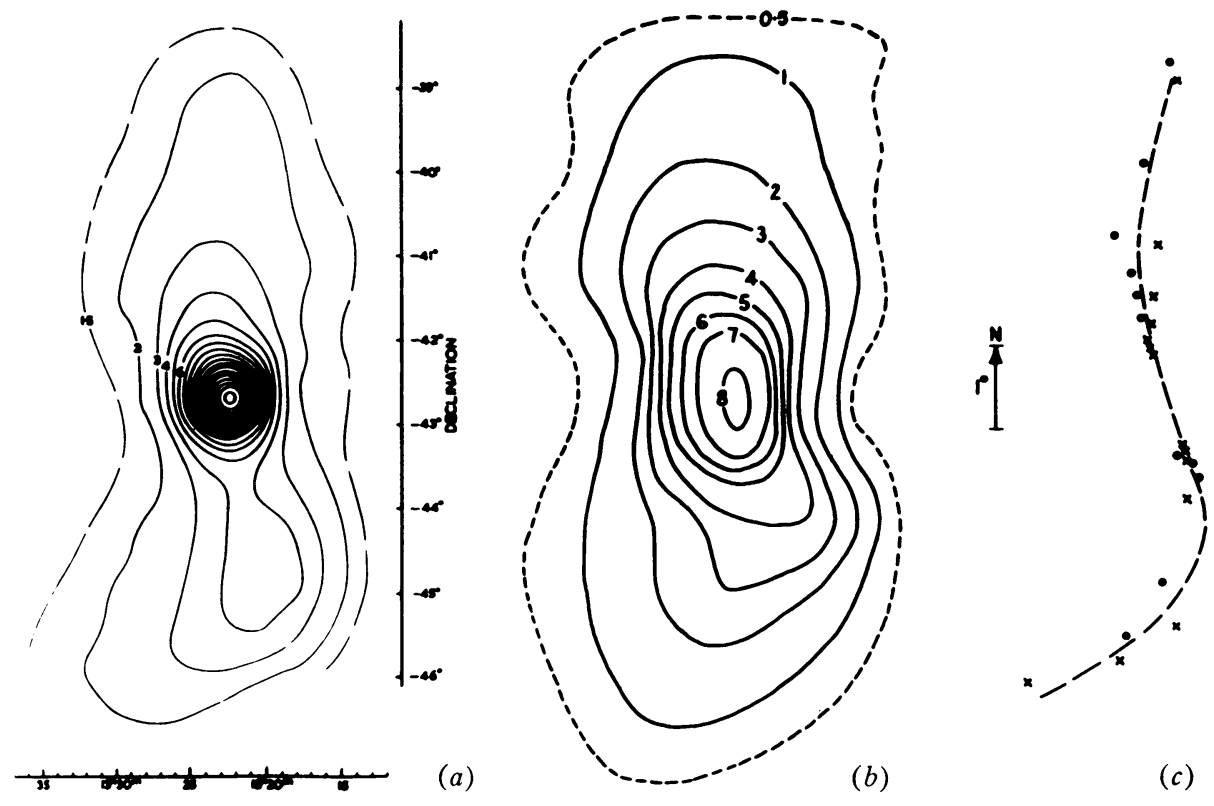

Fig. 1. Centaurus A (a) $85.5 \mathrm{Mc} / \mathrm{s}$, unit $3000^{\circ} \mathrm{K}$; (b) $19.7 \mathrm{Mc} / \mathrm{s}$, unit $100,000^{\circ} \mathrm{K}$; (c) Ridge line; crosses $=85.5-\mathrm{Mc} / \mathrm{s}$ observations; circles $=19.7-\mathrm{Mc} / \mathrm{s}$.

A remarkable feature is the great size of the extended source. At a distance of 750 kiloparsecs (NGC 5128; de Vaucouleurs [4]) the linear dimensions are about 100 kiloparsecs long by 30 kiloparsecs wide. Although it may be objected that the extended source could be some foreground galactic feature superimposed on Centaurus A, no similar features of definite galactic origin have been observed and linear dimensions of similar magnitude are also indicated in the case of Fornax A and Virgo A.

TABLE II

Centaurus a Flux Densities

(Unit: $10^{-24}$ watts $\left.\mathrm{m}^{-2}(\mathrm{c} / \mathrm{s})^{-1}\right)$

$\begin{array}{cccc}\begin{array}{c}\text { Frequency } \\ (\mathrm{Mc} / \mathrm{s})\end{array} & \text { Central Source } & \text { Extended Source } & \text { Total } \\ 19.7 & 30 & 250 & 280 \\ 85.5 & 20 & 67 & 87 \\ 1390 & (2) & (4) & (6)\end{array}$

The various measurements of flux density are collected in Table II. A combination of the $19.7-$ and $85.5-\mathrm{Mc} / \mathrm{s}$ observations with other observations of this source has been given in another paper [3], from which it appears that the source's two components have different spectra (spectral indices of -1.25 and -0.6 for the extended and central sources respectively). The new $1390-\mathrm{Mc} / \mathrm{s}$ data, which provide for the first time a means of definitely dis- 
tinguishing the two components at a high frequency, suggest that the spectral indices are rather closer, but still different. In any case, the $19.7-\mathrm{Mc} / \mathrm{s}$ intensity of the central source, but not of the extended source, is lower than would be expected from extrapolation of the higher frequency intensities.

A study of Figs. 1 $(a)$ and $1(b)$ shows that, even after making allowance for different aerial resolution, the extended source is appreciably broader at 19.7 $\mathrm{Mc} / \mathrm{s}$ than at $85.5 \mathrm{Mc} / \mathrm{s}$. Nevertheless, the maxima of east-west sections fall at the same positions for the two frequencies, although the times of maxima vary with declination. When the ridge line is drawn as in Fig. $1(c)$, it is seen to have a definite spiral shape. The central part of the spiral's apparent position angle is within 10 degrees of the major axis of the main body of NGC 5128 as shown in the long-exposure photograph reproduced by Baade and Minkowski [5]. This might suggest that the extended source is associated with the bright part of the nebula, and the central concentration with the dark band [6], but greater resolving power is required to unravel the complicated structure very near the center. The fact that the dark band and the major axis of the nebula are very nearly at right angles may mean that the shape of the nebula (and of the extended source) is somehow intimately connected with the appearance of the dark band.

In considering the origin of the spiral shape of the extended source, an interesting possibility is that the radiating particles are ejected at high energy from the nebula so that even if there were an equal number of particles of protonic mass the total mass of the extended source would be very small (less than 100 solar masses). The spiral shape would then be a consequence of the nebula's rotation through about one-quarter of a revolution from the start of the ejection, and the age of the extended source would be estimated to be about $10^{7}$ years, the arms expanding at the rate of about $3000 \mathrm{~km} / \mathrm{second}$.

\section{(b) Fornax $A$}

This source is barely resolved by the Sydney cross aerials, but there is no doubt that it, too, is an elongated source, in this case the position angle of the major axis being about 110 degrees. The main observational data are shown in Table III. By taking the distance of the source as 2 megaparsecs (NGC 1316 [4]) we again have a long dimension of the order of 100 kiloparsecs. There is no direct evidence for a single strong central source, but the $85.5-\mathrm{Mc} / \mathrm{s}$ isophotes show definite irregularities, indicative of some kind of fine structure.

\section{TABLE III}

OBSERVATIONS OF Fornax A

$\begin{gathered}\text { Frequency } \\ (\text { Mc/s) }\end{gathered}$
19.7
85.5

\begin{tabular}{cc}
\multicolumn{2}{c}{ Angular } \\
\hline sorth-South & East-West \\
unresolved & 1.1 \\
$\sim 0.5$ & 0.8
\end{tabular}

Flux density
10-24 watts $\mathrm{m}^{-2}(\mathrm{c} / \mathrm{s})^{-1}$
43
9.5

In view of early discussions about the similarity or otherwise of Centaurus 
A and Fornax A the data on the two sources are collected in Table IV. The data in this table tend to support the similarity hypothesis, but this cannot be finally accepted until the optical appearance of NGC 1316 can be reconciled with that of NGC 5128 (for example, by considering the appearance of the latter from a different direction). In particular, the apparent differences in spectra need close study.

\section{TABLE IV}

\section{Comparison Between Centaurus A and fornax A}

\begin{abstract}
Associated galaxy (optical peculiarities)
\end{abstract}

Centaurus A NGC 5128 (heavy absorption band)

Adopted distance (de Vaucouleurs)
Absolute magnitude $(p g)$
Maximum linear dimensions (optical)
Maximum linear dimensions (radio)
Ratio of length to breadth (radio)
Radio frequency spectral index
for whole source
Power radiated between
10 and $3000 \mathrm{Mc} / \mathrm{s}$
Ratio of $85.5 \mathrm{Mc} / \mathrm{s}$ and photo-
graphic flux densities

(c) Virgo $A$

It has been known for some time [6] that at least the greater part of the radio emission from this source originates in a region about 5 minutes of arc in diameter and is associated with the main body of the nebula NGC 4486 . More recently, Baldwin and Smith [7] have given evidence for a faint extended source about 50 minutes of arc in diameter surrounding the small bright source. They concluded from their observations that the extended "halo" was concentric with NGC 4486 .

Observations with the $85.5-\mathrm{Mc} / \mathrm{s}$ cross (Mills, unpublished data) now suggest that this conclusion needs modification. It appears that together with, but not surrounding, the main " point " source there is a much fainter component, extending for between 0.5 and 1.0 on the preceding side. The asymmetrical source is faint, and no detailed brightness distribution has been deduced, but it has been confirmed by other aerial arrangements, and checks on other sources show that instrumental asymmetries are insignificant. The Cambridge positional measurements quoted by Baldwin and Smith are consistent with this asymmetry. At a distance of 9 megaparsecs (Baade and Minkowski [7], corrected), the linear extent of the faint component is of the order of 100 kiloparsecs.

The interest in this observation lies in the fact that the general direction of the extension is the same as that of the "jet" which optical observations have shown issuing from the nucleus of NGC 4486 (see, for example, Baade and Minkowski [7]). Baade and Minkowski's estimate of the jet's age (adjusted for the change in the distance scale) is a few times $10^{6}$ years. If the 
extension to Virgo $A$ is physically associated with the source and particularly with the jet, the expansion must have been extremely rapid, with speeds comparable to those suggested for Centaurus $A$, or must have begun before the start of the jet's ejection.

At $19.7 \mathrm{Mc} / \mathrm{s}$ there is a suggestion of asymmetry on the north-preceding side, but owing to the prevalence of scintillations and the rather low signalto-noise ratio, this is a very doubtful observation.

\section{(d) Large Magellanic Cloud}

A survey of the $19.7-\mathrm{Mc} / \mathrm{s}$ brightness distribution over the region of the sky containing the Large Magellanic Cloud has now been completed, and isophotes of observed brightness temperatures are shown in Fig. 2. Although the area of the sky covered in detail was limited by the observing time available, it appears from a comparison of Fig. 2 and the $85.5-\mathrm{Mc} / \mathrm{s}$ isophotes already published by Mills [8] that the 19.7- and $85.5-\mathrm{Mc} / \mathrm{s}$ emissions cover nearly the same area. The most striking difference between the isophotes at the two frequencies is the different appearance of the $\mathrm{H}$ in emission nebula 30 Doradus; it produces a sharp peak at $85.5 \mathrm{Mc} / \mathrm{s}$, but a dip at $19.7 \mathrm{Mc} / \mathrm{s}$. The significance of this will be discussed below. Another item of interest in Fig. 2 is the dip in brightness temperature centered on $6^{\mathrm{h}} 30^{\mathrm{m}},-68$ degrees, and several degrees in diameter, which is almost certainly due to a galactic $\mathrm{H} \mathrm{II}$ region; it is probably another fragment of Stromlo 12, discussed by Gum [9]. It is

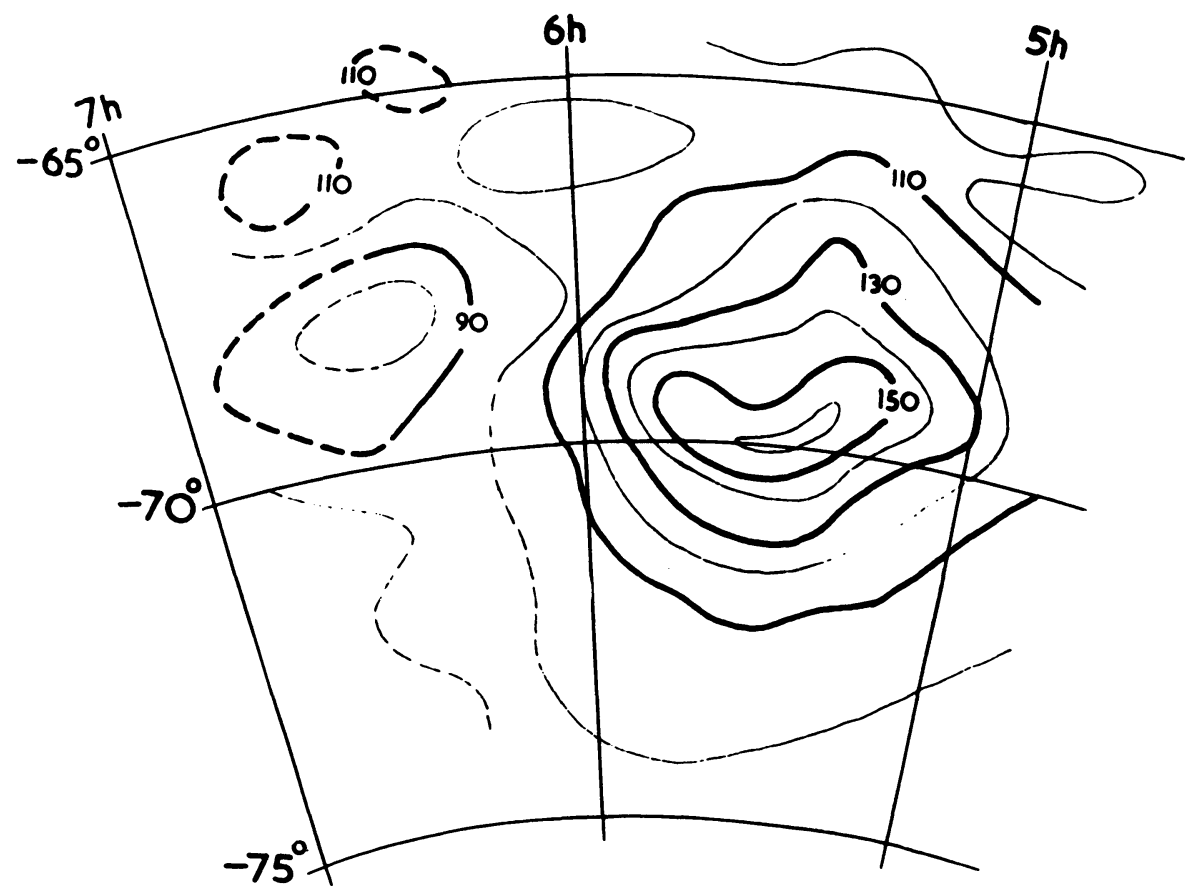

FIG. 2. 19.7-Mc/s isophotes in the region of the Large Magellanic Cloud (unit $=1000^{\circ} \mathrm{K}$ ) 
also worth noting that the isophote of greatest $19.7-\mathrm{Mc} / \mathrm{s}$ brightness temperature follows very nearly the bright "axial bar" seen on photographs of the Large Magellanic Cloud, but this may be coincidence, an effect of the 30 Doradus absorption.

With a source of such extent as the Large Magellanic Cloud, it is difficult to estimate in detail the contribution from the "background" (some of which, of course, is actually foreground emission from the galactic corona), but a first estimate suggests that the ratio of $19.7-$ to $85.5-\mathrm{Mc} / \mathrm{s}$ emission is roughly constant over the Cloud. The ratio of brightness temperatures is about 40 , rather less than the ratio of about 50 observed for galactic radiation away from the galactic plane, but because of the uncertainties involved, the difference may not be significant. The integrated flux density at $19.7 \mathrm{Mc} / \mathrm{s}$ is estimated to be $6 \times 10^{-23}$ watts $\mathrm{m}^{-2}(\mathrm{c} / \mathrm{s})^{-1}$.

The importance of the observation of 30 Doradus in absorption lies in the fact that the absorbing region is well removed from the Galaxy; the radiation that is absorbed comes only from the Large Magellanic Cloud itself and from the integrated effect of all other extragalactic sources in the line of sight. Since the former component can be estimated from the observations, the latter can, at least in principle, be deduced, which leads to the possibility of separating the galactic and extragalactic contributions to high-latitude temperatures.

The situation is illustrated in Fig. 3. It is assumed that the contribution to aerial temperatures from the galactic corona, $T_{g}$, and from the "universe," $T_{u}$, are constant over the few degrees around 30 Doradus. $T_{c}$ is the contribution to be expected, if there were no absorption, from the Large Magellanic

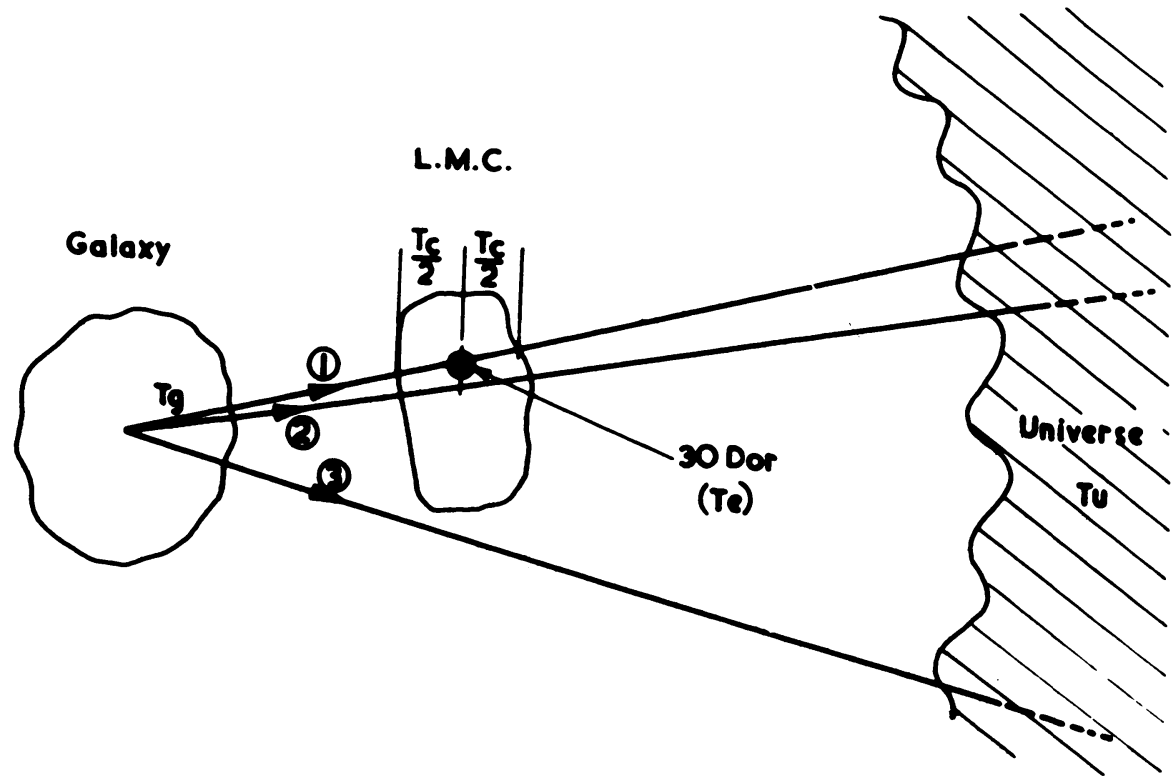

FIG. 3. Illustrating the absorption of extragalactic radiation in 30 Doradus. 
Cloud in the direction of 30 Doradus, and $T_{\theta}$ is the electron temperature in 30 Doradus itself, which is assumed to be in the center of the line of sight through the Large Magellanic Cloud. $\alpha$ is the fraction of radiation in the aerial beam which is absorbed in 30 Doradus, taking into account the size of the beam. Then, if $T_{1}$ is the temperature actually observed in the direction of 30 Doradus and $T_{2}$ that which would be observed in the absence of absorption, $\left(T_{2}-T_{1}\right)=\alpha T_{u}+\frac{1}{2} \alpha T_{c}-\alpha T_{e}$, from which $T_{u}=\left(T_{2}-T_{1}\right) / \alpha+T_{e}-\frac{1}{2} T_{c}$. From the observations we estimate that $T_{0}=84,000^{\circ} \mathrm{K}$ and $\left(T_{2}-T_{1}\right)=$ $20,000^{\circ} \mathrm{K}$. Taking $T_{e}=10,000^{\circ} \mathrm{K}$, we then have $T_{u}=20,000 / \alpha-32,000^{\circ} \mathrm{K}$. At present the value of $\alpha$ can only be estimated from optical data, and unfortunately these are not readily available in a suitable form. The most useful information is contained in Henize's catalogue [10]. Taken in conjunction with the photometric data of Doherty, Henize, and Aller [11], it would appear that absorption of $19.7 \cdot \mathrm{Mc} / \mathrm{s}$ radiation is effectively complete in an $\mathrm{H}$ in region of Henize brightness 2 or higher. However, the $\mathrm{H}$ II region numbered $\mathrm{N} 135$, the largest in angular size and therefore occupying the largest fraction of the aerial beam, has patchy emission, and it is not clear whether its brightness " 2 " refers to an average, or only to some of the features. If the whole of $\mathrm{N} 135$ is taken as completely absorbing, the value of $\alpha$ is 0.52 ; a minimum value of 0.21 is found by taking only the bright compact $\mathrm{H}$ II regions and neglecting $\mathrm{N} 135$. As a conservative estimate we could say that $\mathrm{N} 135$ absorbs one-half of the incident radiation that is not cut off by the bright regions, and take $\alpha=0.37$; then $T_{u}=22,000^{\circ} \mathrm{K}$. If $\alpha$ is taken as $0.52, T_{u}=$ $7000^{\circ} \mathrm{K}$. In the vicinity of the Large Magellanic Cloud the non-Cloud radiation gives a brightness temperature of about $80,000^{\circ} \mathrm{K}$, so that here the galactic corona must produce more than two-thirds, perhaps 90 per cent, of the emission. The minimum brightness temperature observed is about $50,000^{\circ} \mathrm{K}$, of which the Galaxy must contribute more than half, and possibly over 80 per cent.

It is hoped that photoelectric observations being undertaken at Mount Stromlo will eventually reduce the uncertainty in the value of $\alpha$.

\section{(e) Small Magellanic Cloud}

Because of the lower brightness, the Small Magellanic Cloud is more difficult to observe. It has been clearly detected at $19.7 \mathrm{Mc} / \mathrm{s}$ but the analysis of the records is not yet complete. All that can be said at present is that the general picture at $19.7 \mathrm{Mc} / \mathrm{s}$ appears to be much the same as at $85.5 \mathrm{Mc} / \mathrm{s}$ [8], including the possibility of a similar extension toward the Large Magellanic Cloud. The ratio of brightness temperatures is about the same as for the Large Magellanic Cloud, but no attempt has been made to determine an integrated flux density.

\section{DISCUSSION}

We shall briefly consider several points arising out of the observational results.

(1) The three "radio galaxies" observed appear to have angular sizes 
much greater than the associated optical galaxies have. In each case the source's linear dimensions are of the order of 100 kiloparsecs. On the other hand, the radio emission (at least in the continuum) from the Magellanic Clouds is largely contained within the optical limits. Therefore, $(a)$ when angular sizes of a number of radio sources are determined, any optical search for associated galaxies must extend to galaxies having diameters down to one-tenth or less of the radio diameters, and $(b)$ if the corona of our Galaxy is similar to that of the Large Magellanic Cloud, its radius might be expected to be only 10 or 12 kiloparsecs.

(2) The comparatively low brightness-temperature estimate of the integrated emission of all the external galaxies will be of importance in checking any cosmological hypotheses that explain observed number-intensity distributions of discrete sources. In view of all the uncertainties of the data, both radio and optical, it seems profitless to go into the matter in detail at this stage. It does seem, however, that only a small part of the extragalactic radiation comes from normal galaxies. The three " radio galaxies" considered in this paper show some similarities, and the radio observations increase interest in Ambartsumian's suggestion of a class of dividing rather than of colliding galaxies. NGC 5128 (Centaurus A) cannot be a typical " radio galaxy" for, if it is, it can be shown that the space density required to account for all the extragalactic emission is of the order of $10^{-20} / \mathrm{parsec}^{3}$, which is probably rather too high to agree with the number of moderately bright sources observed. Any such estimate of the space density would require considerable modification if it can be shown definitely that the ages of the sources of emission are only of the order of $10^{7}$ years.

The author is indebted to a number of his colleagues for helpful discussions, and for permission to quote unpublished data.

\section{REFERENCES}

[1] de Vaucouleurs, G., and Sheridan, K. V. Radio Astronomy (I.A.U. Symposium No. 4, 1955). Cambridge, England, 1957, p. 169.

[2] Sheridan, K. V. Aust. J. Phys. 11, 400, 1958.

[3] Shain, C. A. To be published in Aust. J. Phys. December 1958.

[4] de Vaucouleurs, G. Occ. Notes R. Astr. Soc. 3, 118, 1956.

[5] Baade, W., and Minkowski, R. Ap. J. 119, 215, 1954.

[6] Mills, B. Y. Aust. J. Phys. 6, 452, 1953.

[7] Baldwin, J. E., and Smith, F. G. The Observatory, 76, 141, 1956.

[8] Mills, B. Y. Aust. J. Phys. 8, 368, 1955. (For a correction to scale of brightness temperature see article by Mills in Handbuch der Physik, Vol. 53.)

[9] Gum, C. S. The Observatory, 76, 150, 1956.

[10] Henize, K. G. Ap. J. Suppl. 2, 315, 1956.

[11] Doherty, L., Henize, K. G., and Aller, L. H. Ap. J. Suppl. 2, 345, 1956.

\section{Discussion}

Minkowski: In connection with the asymmetry of the Virgo $A$ source it seems of interest to mention the fact that the velocity of the $[\mathrm{O} \mathrm{II}]$ line $\lambda 3727$ 
in the nucleus differs from that of the stellar absorption lines. This was first observed by Humason many years ago. The observations have now been repeated and confirmed with much higher dispersion. The velocity of $\lambda 3727$, which is a broad and asymmetrical line, differs by $-225 \mathrm{~km} / \mathrm{second}$ from the stellar velocities in the nucleus. This seems to indicate that gas is streaming out of or through the nucleus.

Smith: Could we have some further details of the measurements of the asymmetry of $M 87$, so that a comparison may be made with previous observations of the halo?

Mills: The main evidence for asymmetry is a direct observation with the $85.5-\mathrm{Mc} / \mathrm{s}$ fan-beam system (EW beamwidth 0.6 degrees). This gives a small but quite definite extension on the preceding side. This picture is also suggested by the less reliable pencil-beam observations at 85.5 and $19.7 \mathrm{Mc} / \mathrm{s}$. 